\title{
The Number of Canalyzing Functions over Any Finite Set ${ }^{*}$
}

\author{
Yuan Li ${ }^{1 \#}$, David Murrugarra ${ }^{2}$, John O. Adeyeye ${ }^{1}$, Reinhard Laubenbacher ${ }^{3}$ \\ ${ }^{1}$ Department of Mathematics, Winston-Salem State University, Winston-Salem, USA \\ ${ }^{2}$ School of Mathematics, Georgia Tech, Atlanta, USA \\ ${ }^{3}$ Virginia Bioinformatics Institute, Virginia Tech, Blacksburg, USA \\ Email: "liyu@wssu.edu
}

Received March 1, 2013; revised May 20, 2013; accepted June 16, 2013

Copyright (C) 2013 Yuan Li et al. This is an open access article distributed under the Creative Commons Attribution License, which permits unrestricted use, distribution, and reproduction in any medium, provided the original work is properly cited.

\begin{abstract}
In this paper, we extend the definition of Boolean canalyzing functions to the canalyzing functions of multi-state case. Namely, $f: Q^{n} \rightarrow Q$, where $Q=\left\{a_{1}, a_{2}, \cdots, a_{q}\right\}$. We obtain its cardinality and the cardinalities of its various subsets (They may not be disjoint). When $q=2$, we obtain a combinatorial identity by equating our result to the formula in [1]. For a better understanding to the magnitude, we obtain the asymptotes for all the cardinalities as either $n \rightarrow \infty$ or $q \rightarrow \infty$.
\end{abstract}

Keywords: Canalyzing Function; Inclusion and Exclusion Principle

\section{Introduction}

The idea of canalization was initiated from Waddington, C. H. [2]. When comparing the class of canalyzing functions to other classes of functions with respect to their evolutionary plausibility as emergent control rules in genetic regulatory systems, it is informative to know the number of canalyzing functions with a given number of input variables [1]. However, the Boolean network modeling paradigm is rather restrictive, with its limit to two possible functional levels, ON and OFF, for genes, proteins, etc. Many discrete models of biological networks therefore allow variables to take on multiple states. Common used discrete multi-state model types are socalled logical models [3], Petri nets [4], and agent- based models [5].

In this paper, we generalize the concept of Boolean canalyzing rules to the multi-state case. By generalizing the results in [1], we provide formulas for the cardinalities of various subsets of canalyzing functions. We also obtain the asymptotes of these cardinalities as either $n \rightarrow \infty$ or $q \rightarrow \infty$. We obtain a combinatorial identity by equating our result to the formula in [1].

\section{Preliminaries}

In this section we introduce the definition of a canalyzing

*Supported by an award from the USA DoD \# W911NF-11-10166.

\#Corresponding author. function.

Let $[n]=\{1,2, \cdots, n\}, Q=\left\{a_{1}, a_{2}, \cdots, a_{q}\right\}$ and $f: Q^{n} \rightarrow Q$.

A function is canalyzing if there is a variable $x_{i}$ and an element $a \in Q$ so that the value of the function is fixed once variable $x_{i}$ is fixed at $a$. More precisely, we have the following definitions.

Definition 2.1

1) The function $f\left(x_{1}, x_{2}, \cdots, x_{n}\right)$ is $\langle i: a: b\rangle$ canalyzing if $f\left(x_{1}, \cdots, x_{i-1}, a, x_{i+1}, \cdots, x_{n}\right)=b$, for all $x_{1}, \cdots, x_{i-1}, x_{i+1}, \cdots, x_{n}$.

2) The function $f\left(x_{1}, x_{2}, \cdots, x_{n}\right)$ is $\langle i: a:-\rangle$ canalyzing if there exists $b \in Q$ such that $f\left(x_{1}, \cdots, x_{i-1}, a, x_{i+1}, \cdots, x_{n}\right)=b$, for all $x_{1}, \cdots, x_{i-1}, x_{i+1}, \cdots, x_{n}$.

3) The function $f\left(x_{1}, x_{2}, \cdots, x_{n}\right)$ is $\langle-: a: b\rangle$ canalyzing if there exists $i \in[n]$ such that

$f\left(x_{1}, \cdots, x_{i-1}, a, x_{i+1}, \cdots, x_{n}\right)=b$, for all

$x_{1}, \cdots, x_{i-1}, x_{i+1}, \cdots, x_{n}$.

4) The function $f\left(x_{1}, x_{2}, \cdots, x_{n}\right)$ is $\langle i:-: b\rangle$ canalyzing if there exists $a \in Q$ such that $f\left(x_{1}, \cdots, x_{i-1}, a, x_{i+1}, \cdots, x_{n}\right)=b$, for all $x_{1}, \cdots, x_{i-1}, x_{i+1}, \cdots, x_{n}$.

5) The function $f\left(x_{1}, x_{2}, \cdots, x_{n}\right)$ is $\langle-: a:-\rangle$ canalyzing if there exist $i \in[n], b \in Q$ such that $f\left(x_{1}, \cdots, x_{i-1}, a, x_{i+1}, \cdots, x_{n}\right)=b$, for all $x_{1}, \cdots, x_{i-1}, x_{i+1}, \cdots, x_{n}$.

6) The function $f\left(x_{1}, x_{2}, \cdots, x_{n}\right)$ is $\langle i:-:-\rangle$ 
canalyzing if there exist $a, b \in Q$ such that

$f\left(x_{1}, \cdots, x_{i-1}, a, x_{i+1}, \cdots, x_{n}\right)=b$, for all

$x_{1}, \cdots, x_{i-1}, x_{i+1}, \cdots, x_{n}$.

7) The function $f\left(x_{1}, x_{2}, \cdots, x_{n}\right)$ is $\langle-:-: b\rangle$ canalyzing if there exist $i \in[n], a \in Q$ such that

$f\left(x_{1}, \cdots, x_{i-1}, a, x_{i+1}, \cdots, x_{n}\right)=b$, for all

$x_{1}, \cdots, x_{i-1}, x_{i+1}, \cdots, x_{n}$.

8) The function is $f\left(x_{1}, x_{2}, \cdots, x_{n}\right)$ is $\langle-:-:-\rangle$ canalyzing if there exist $i \in[n], a, b \in Q$ such that

$f\left(x_{1}, \cdots, x_{i-1}, a, x_{i+1}, \cdots, x_{n}\right)=b$, for all

$x_{1}, \cdots, x_{i-1}, x_{i+1}, \cdots, x_{n}$.

By abuse of notation, we also use $\langle i:-: b\rangle$ to stand for the set of all the $\langle i:-: b\rangle$ canalyzing functions, $\langle i: a: b\rangle$ will stand for the set of all the $\langle i: a: b\rangle$ canalyzing functions and etc. We use $\phi$ to stand for the empty set.

By the definitions, we immediately have the following propositions.

Proposition 2.2 If $b_{1} \neq b_{2}$, then

$\left\langle i: a: b_{1}\right\rangle \cap\left\langle i: a: b_{2}\right\rangle=\phi$.

Proposition 2.3 If $b_{1} \neq b_{2}$ and $i_{1} \neq i_{2}$, then

$\left\langle i_{1}:-: b_{1}\right\rangle \cap\left\langle i_{2}:-: b_{2}\right\rangle=\phi$.

By the definitions, we have

$$
\begin{aligned}
& \langle-:-:-\rangle=\bigcup_{b \in Q}\langle-:-: b\rangle=\bigcup_{a \in Q}\langle-: a:-\rangle=\bigcup_{i \in[n]}\langle i:-:-\rangle, \\
& \langle-:-: b\rangle=\bigcup_{i \in[n]}\langle i:-: b\rangle=\bigcup_{a \in Q}\langle-: a: b\rangle, \\
& \langle-: a:-\rangle=\bigcup_{b \in Q}\langle-: a: b\rangle=\bigcup_{i \in[n]}\langle i: a:-\rangle, \\
& \langle i:-:-\rangle=\bigcup_{a \in Q}\langle i: a:-\rangle=\bigcup_{b \in Q}\langle i:-: b\rangle, \\
& \langle i:-: b\rangle=\bigcup_{a \in Q}\langle i: a: b\rangle, \\
& \langle i: a:-\rangle=\bigcup_{b \in Q}\langle i: a: b\rangle, \\
& \langle-: a: b\rangle=\bigcup_{i \in[n]}\langle i: a: b\rangle .
\end{aligned}
$$

For any set $S$, we use $|S|$ to stand for its cardinality. We use $C(n, k)=\frac{n !}{k !(n-k) !}$ to stand for the binomial coefficients. As usual, $C(n, k)$ should be explained as zero once $k>n$.

Obviously, for the above notations, the cardinality are same for different values of $i, a$ and $b$. In other words, we have $\left|\left\langle i_{1}: a_{1}: b_{1}\right\rangle\right|=\left|\left\langle i_{2}: a_{2}: b_{2}\right\rangle\right|$, $|\langle i:-: b\rangle|=|\langle j:-: c\rangle|,|\langle-: a: b\rangle|=|\langle-: c: d\rangle|$ and etc.

\section{Enumeration}

Theorem 3.1 Given $i \in[n], a, b \in Q$, the number of $\langle i: a: b\rangle$ canalyzing functions is $q^{q^{n}-q^{n-1}}$. In other words, we have $|\langle i: a: b\rangle|=q^{q^{n}-q^{n-1}}$.
Proof: A function in the set $\langle i: a: b\rangle$ is uniquely determined by its value on inputs $\left(x_{1}, \cdots, x_{n}\right)$ with $x_{i} \neq a$. There are $(q-1) q^{n-1}=q^{n}-q^{n-1}$ such inputs, and the function can take $q$ different values. Thus $|\langle i: a: b\rangle|=q^{q^{n}-q^{n-1}}$. 口

Because $\langle i: a:-\rangle=\bigcup_{b \in Q}\langle i: a: b\rangle$, by Proposition 2.2, we get

Theorem 3.2 The number of all the $\langle i: a:-\rangle$ canalyzing function is

$$
q\left(q^{q^{n}-q^{n-1}}\right)=q^{q^{n}-q^{n-1}+1} .
$$

Lemma 3.3 We have $\left|\bigcap_{j=1}^{k}\left\langle i: a_{j}: b\right\rangle\right|=q^{q^{n}-k q^{n-1}}$ for any $\left\{a_{1}, a_{2}, \cdots, a_{k}\right\} \subset Q$.

Proof: A function in the set $\bigcap_{j=1}^{k}\left\langle i: a_{j}: b\right\rangle$ is uni quely determined by it values on inputs $\left(x_{1}, \cdots, x_{n}\right)$ with $x_{i} \notin\left\{a_{1}, \cdots, a_{k}\right\}$. There are $(q-k) q^{n-1}=q^{n}-k q^{n-1}$ such inputs. $\square$

Theorem 3.4 Given $i \in[n]$ and $b \in Q$, the number of $\langle i:-: b\rangle$ canalyzing functions is $q^{q^{n}}-\left(q^{q^{n-1}}-1\right)^{q}$. In other words, we have $|\langle i:-: b\rangle|=q^{q^{n}}-\left(q^{q^{n-1}}-1\right)^{q}$.

Proof: By Inclusion and Exclusion Principle, we have

$$
\begin{aligned}
& |\langle i:-: b\rangle|=\left|\bigcup_{a \in Q}\langle i: a: b\rangle\right| \\
& =\bigcup_{a \in Q}|\langle i: a: b\rangle|-\sum_{\left\{a_{1}, a_{2}\right\} \subset Q}\left|\left\langle i: a_{1}: b\right\rangle \cap\left\langle i: a_{2}: b\right\rangle\right| \\
& +\cdots+(-1)^{k-1} \sum_{\left\{a_{1}, a_{2}, \cdots, a_{k}\right\} \subset Q}\left|\bigcap_{j=1}^{k}\left\langle i: a_{j}: b\right\rangle\right|+\cdots+(-1)^{q-1} \\
& =C(q, 1) q^{q^{n}-q^{n-1}}-C(q, 2) q^{q^{n}-2 q^{n-1}} \\
& +\cdots+(-1)^{k-1} C(q, k) q^{q^{n}-k q^{n-1}}+\cdots+(-1)^{q-1} \\
& =\sum_{k=1}^{q}(-1)^{k-1} C(q, k) q^{q^{n}-k q^{n-1}} \\
& =q^{q^{n}} \sum_{k=1}^{q}\left(-C(q, k)\left(-q^{-q^{n-1}}\right)^{k}\right) \\
& =q^{q^{n}}\left(1-\left(1-q^{-q^{n-1}}\right)^{q}\right)=q^{q^{n}}-\left(q^{q^{n-1}}-1\right)^{q} \text {. }
\end{aligned}
$$

Similar to Lemma 3.3, we have

Lemma 3.5 If $\left\{i_{1}, i_{2}, \cdots, i_{k}\right\} \subset[n]$, then

$\left|\bigcap_{j=1}^{k}\left\langle i_{j}: a: b\right\rangle\right|=q^{(q-1)^{k} q^{n-k}}$.

Based on this lemma, we can get the following result. Theorem 3.6 We have

$$
|\langle-: a: b\rangle|=\sum_{1 \leq k \leq n}(-1)^{k-1} C(n, k) q^{(q-1)^{k} q^{n-k}} .
$$

Proof: By Inclusion and Exclusion Principle, we have 


$$
\begin{aligned}
|\langle-: a: b\rangle|=\left|\bigcup_{i \in[n]}\langle i: a: b\rangle\right| \\
=\sum_{1 \leq i \leq n}|\langle i: a: b\rangle|-\sum_{1 \leq i<j \leq n}|\langle i: a: b\rangle \cap\langle j: a: b\rangle| \\
+\cdots+(-1)^{k-1} \\
\quad \cdot \sum_{1 \leq i_{1}<\cdots<i_{k} \leq n} \bigcap_{j=1}^{k}\left\langle i_{j}: a: b\right\rangle\left|+\cdots+(-1)^{n-1}\right| \bigcap_{j=1}^{n}\langle j: a: b\rangle \mid \\
=C(n, 1) q^{(q-1) q^{n-1}-C(n, 2) q^{(q-1)^{2}} q^{n-2}} \\
+\cdots+(-1)^{k-1} C(n, k) q^{(q-1)^{k} q^{n-k}}+\cdots+(-1)^{n-1} q^{(q-1)^{n}} \\
=\sum_{1 \leq k \leq n}(-1)^{k-1} C(n, k) q^{(q-1)^{k} q^{n-k}} .
\end{aligned}
$$

From the above theorem, we can get the following result.

Theorem 3.7 We have

$$
|\langle-: a:-\rangle|=q \sum_{1 \leq k \leq n}(-1)^{k-1} C(n, k) q^{(q-1)^{k} q^{n-k}}
$$

Proof: Because $\langle-: a:-\rangle=\bigcup_{b \in Q}\langle-: a: b\rangle$, by Theorem 3.6 , we just need to show $\left\langle-: a: b_{1}\right\rangle \cap\left\langle-: a: b_{2}\right\rangle=\phi$ if $b_{1} \neq b_{2}$. Suppose $f \in\left\langle-: a: b_{1}\right\rangle \cap\left\langle-: a: b_{2}\right\rangle$, then there exist $i_{1}$ and $i_{2} \in[n]$ such that

$$
f \in\left\langle i_{1}: a: b_{1}\right\rangle \cap\left\langle i_{2}: a: b_{2}\right\rangle \text { since }\langle-: a: b\rangle=\bigcup_{i \in[n]}\langle i: a: b\rangle \text {. }
$$

If $i_{1}=i_{2}$, we get a contradiction by Proposition 2.2. If $i_{1} \neq i_{2}$, we get a contradiction by Proposition 2.3 since $\left\langle i_{1}: a: b_{1}\right\rangle \subset\left\langle i_{1}:-: b_{1}\right\rangle$ and $\left\langle i_{2}: a: b_{2}\right\rangle \subset\left\langle i_{2}:-: b_{2}\right\rangle$.

Now, we are going to find the formula for the number of all the canalyzing functions with given canalyzed value $b$. In other words, the formula of $|\langle-:-: b\rangle|$.

Let $S_{b}=\{\langle i: a: b\rangle \mid i \in[n], a \in Q\}$ for any $b \in Q$. By Inclusion and Exclusion Principle, we have

$$
|\langle-:-: b\rangle|=\left|\bigcup_{i \in[n]} \bigcup_{a \in Q}\langle i: a: b\rangle\right|=\sum_{k=1}^{n q}(-1)^{k-1} N_{k} .
$$

where

$$
N_{k}=\sum_{s \subset S_{b},|s|=k}\left|\bigcap_{T \in S} T\right| .
$$

In order to evaluate $N_{k}$, we write all the members in $S_{b}$ as the following $n \times q$ matrix.

$$
A=\left(\begin{array}{cccc}
\left\langle 1: a_{1}: b\right\rangle & \left\langle 1: a_{2}: b\right\rangle & \cdots & \left\langle 1: a_{q}: b\right\rangle \\
\left\langle 2: a_{1}: b\right\rangle & \left\langle 2: a_{2}: b\right\rangle & \cdots & \left\langle 2: a_{q}: b\right\rangle \\
\vdots & \vdots & \ddots & \vdots \\
\left\langle n: a_{1}: b\right\rangle & \left\langle n: a_{2}: b\right\rangle & \cdots & \left\langle n: a_{q}: b\right\rangle
\end{array}\right)
$$

For any $s \subset S_{b}$ with $|s|=k$, we will choose $k$ ele- ments from the above matrix to form $s$.

Suppose $k_{1}$ of its elements are from the first row (there are $C\left(q, k_{1}\right)$ ways to do so). Let these $k_{1}$ elements be $\left\langle 1: a_{11}: b\right\rangle,\left\langle 1: a_{12}: b\right\rangle, \cdots,\left\langle 1: a_{1 k_{1}}: b\right\rangle$.

Suppose $k_{2}$ of its elements are from the second row (there are $C\left(q, k_{2}\right)$ ways to do so). Let these $k_{2}$ elements be $\left\langle 2: a_{21}: b\right\rangle,\left\langle 2: a_{22}: b\right\rangle, \cdots,\left\langle 2: a_{2 k_{2}}: b\right\rangle$.

Suppose $k_{n}$ of its elements are from the last row (there are $C\left(q, k_{n}\right)$ ways to do so). Let these $k_{n}$ elements be $\left\langle n: a_{n 1}: b\right\rangle,\left\langle n: a_{n 2}: b\right\rangle, \cdots,\left\langle n: a_{n k_{n}}: b\right\rangle$.

$$
k_{1}+k_{2}+\cdots+k_{n}=k, 0 \leq k_{i} \leq q, i=1,2, \cdots, n .
$$

Similar to Lemma 3.3, we have

Lemma 3.8 Let $s$ be the subset of $S_{b}$ as mentioned above, then $\left|\bigcap_{T \in S} T\right|=q^{\left(q-k_{1}\right)\left(q-k_{2}\right) \cdots\left(q-k_{n}\right)}$.

Hence,

$$
N_{k}=\sum_{k_{1}+\cdots+k_{n}=k} C\left(q, k_{1}\right) \cdots C\left(q, k_{n}\right) q^{\left(q-k_{1}\right) \cdots\left(q-k_{n}\right)} .
$$

We get

Theorem 3.9 For any $b \in Q$, we have

$$
\begin{aligned}
& |\langle-:-: b\rangle| \\
& =\sum_{k=1}^{n q}(-1)^{k-1}\left(\sum_{k_{1}+k_{2}+\cdots+k_{n}=k}\left(\prod_{j=1}^{n} C\left(q, k_{j}\right)\right) q^{\prod_{j=1}^{n}\left(q-k_{j}\right)}\right) .
\end{aligned}
$$

In order to evaluate $\langle i:-:-\rangle$, we need two more lemmas. Their proofs are similar to that of Lemma 3.3 and we omit them.

Lemma 3.10 If $\left\{a_{1}, a_{2}, \cdots, a_{k}\right\} \subset Q$ and $\left\{b_{1}, b_{2}, \cdots, b_{k}\right\} \subset Q$, then

$$
\left|\bigcap_{j=1}^{k}\left\langle i: a_{j}: b_{j}\right\rangle\right|=q^{(q-k) q^{n-1}} .
$$

Lemma 3.11 If $a_{11}, \cdots, a_{1 k_{1}} ; a_{21}, \cdots, a_{2 k_{2}} ; \cdots ; a_{r 1}, \cdots, a_{r k_{r}}$ are $k_{1}+\cdots+k_{r}$ distinct elements of $Q$, $\left\{b_{1}, \cdots, b_{r}\right\} \subset Q$. Then,

$$
\begin{aligned}
& \left|\left(\bigcap_{j=1}^{k_{1}}\left\langle i: a_{1 j}: b_{1}\right\rangle\right) \cap\left(\bigcap_{j=1}^{k_{2}}\left\langle i: a_{2 j}: b_{2}\right\rangle\right) \cdots \cap\left(\bigcap_{j=1}^{k_{r}}\left\langle i: a_{r j}: b_{r}\right\rangle\right)\right| \\
& =q^{\left(q-k_{1}-k_{2}-\cdots-k_{r}\right) q^{n-1}}
\end{aligned}
$$

Now, we are ready to find the cardinality of $\langle i:-:-\rangle$. Theorem 3.12 We have

$$
\begin{aligned}
& |\langle i:-:-\rangle| \\
& =q ! \sum_{k=1}^{q} \frac{(-1)^{k-1} q^{(q-k) q^{n-1}}}{(q-k) !}\left(\sum_{k_{1}+\cdots+k_{q}=k, 0 \leq k_{i} \leq q} \frac{1}{k_{1} ! k_{2} ! \cdots k_{q} !}\right) .
\end{aligned}
$$

Proof: First, we have $\langle i:-:-\rangle=\bigcup_{a \in Q} \bigcup_{b \in Q}\langle i: a: b\rangle$. 
Let $S_{i}=\{\langle i: a: b\rangle \mid a, b \in Q\}$, we get

$|\langle i:-:-\rangle|=\sum_{k=1}^{q^{2}}(-1)^{k-1} N_{k}$. Where

$$
N_{k}=\sum_{s \in S_{i}, s \mid=k}\left|\bigcap_{T \in s} T\right| .
$$

In order to evaluate $N_{k}$, we write all the elements in $S_{i}$ as the following $q \times q$ matrix.

$$
B=\left(\begin{array}{cccc}
\left\langle i: a_{1}: b_{1}\right\rangle & \left\langle i: a_{2}: b_{1}\right\rangle & \cdots & \left\langle i: a_{q}: b_{1}\right\rangle \\
\left\langle i: a_{1}: b_{2}\right\rangle & \left\langle i: a_{2}: b_{2}\right\rangle & \cdots & \left\langle i: a_{q}: b_{2}\right\rangle \\
\vdots & \vdots & \ddots & \vdots \\
\left\langle i: a_{1}: b_{q}\right\rangle & \left\langle i: a_{2}: b_{q}\right\rangle & \cdots & \left\langle i: a_{q}: b_{q}\right\rangle
\end{array}\right)
$$

For any $s \subset S_{i}$ with $|s|=k$, we will choose $k$ elements from the above matrix to form $s$.

Suppose $k_{1}$ of it elements are from the first row (There are $C\left(q, k_{1}\right)$ ways to do so). Let these $k_{1}$ elements be $\left\langle i: a_{11}: b_{1}\right\rangle,\left\langle i: a_{12}: b_{1}\right\rangle, \cdots,\left\langle i: a_{1 k_{1}}: b_{1}\right\rangle$.

Suppose $k_{2}$ of its elements are from the second row, we must choose these elements from different columns, otherwise the intersection will be $\phi$ by Proposition 2.2 (There are $C\left(q-k_{1}, k_{2}\right)$ ways to do so). Let these $k_{2}$ elements be $\left\langle i: a_{21}: b_{2}\right\rangle,\left\langle i: a_{22}: b_{2}\right\rangle, \cdots,\left\langle i: a_{2 k_{2}}: b_{2}\right\rangle$

Suppose $k_{q}$ of its elements are from the last row (There are $C\left(q-k_{1}-k_{2}-\cdots-k_{q-1}, k_{q}\right)$ ways to do so). Let these $k_{q}$ elements be

$$
\left\langle i: a_{q 1}: b_{q}\right\rangle,\left\langle i: a_{q 2}: b_{q}\right\rangle, \cdots,\left\langle i: a_{q k_{q}}: b_{q}\right\rangle \text {. }
$$

where $k_{1}+k_{2}+\cdots+k_{q}=k, 0 \leq k_{i} \leq q, i=1,2, \cdots, q$. We have

$$
\begin{aligned}
N_{k}= & \sum_{s \in S_{i},|s|=k}\left|\bigcap_{T \in S} T\right| \\
= & \sum_{k_{1}+\cdots+k_{q}=k, 0 \leq k_{i} \leq q} C\left(q, k_{1}\right) C\left(q-k_{1}, k_{2}\right) \\
& \cdots C\left(q-k_{1}-\cdots-k_{q-1}, k_{q}\right) I_{k_{1} k_{2} \cdots k_{q}}
\end{aligned}
$$

where

$$
\begin{aligned}
& I_{k_{1} k_{2} \cdots k_{q}}= \\
& \left|\left(\bigcap_{j=1}^{k_{1}}\left\langle i: a_{1 j}: b_{1}\right\rangle\right) \cap\left(\bigcap_{j=1}^{k_{2}}\left\langle i: a_{2 j}: b_{2}\right\rangle\right) \cdots \cap\left(\bigcap_{j=1}^{k_{q}}\left\langle i: a_{q j}: b_{q}\right\rangle\right)\right|
\end{aligned}
$$

By Lemma 3.11, we know

$$
I_{k_{1} k_{2} \cdots k_{q}}=q^{\left(q-k_{1}-k_{2} \cdots \cdots-k_{q}\right) q^{n-1}}=q^{(q-k) q^{n-1}} .
$$

This number is zero if $k>q$.

A straightforward computing shows that

$$
\begin{aligned}
& C\left(q, k_{1}\right) C\left(q-k_{1}, k_{2}\right) \cdots C\left(q-k_{1}-\cdots-k_{q-1}, k_{q}\right) \\
& =\frac{q !}{k_{1} ! k_{2} ! \cdots k_{q} !(q-k) !} .
\end{aligned}
$$

Hence, we get

$$
\begin{aligned}
& |\langle i:-:-\rangle| \\
& =\sum_{k=1}^{q^{2}}(-1)^{k-1} N_{k}=\sum_{k=1}^{q}(-1)^{k-1} N_{k} \\
& =\sum_{k=1}^{q}(-1)^{k-1}\left(\sum_{k_{1}+\cdots+k_{q}=k, 0 \leq k_{i} \leq q} \frac{q !}{k_{1} ! k_{2} ! \cdots k_{q} !(q-k) !} q^{(q-k) q^{n-1}}\right) \\
& =q ! \sum_{k=1}^{q} \frac{(-1)^{k-1} q^{(q-k) q^{n-1}}}{(q-k) !} \sum_{k_{1}+\cdots+k_{q}=k, 0 \leq k_{i} \leq q} \frac{1}{k_{1} ! k_{2} ! \cdots k_{q} !} .
\end{aligned}
$$

Now we begin to evaluate $|\langle-:-:-\rangle|$.

Theorem 3.13 We have

$$
\begin{aligned}
& |\langle-:-:-\rangle| \\
& =\sum_{k=1}^{q}(-1)^{k-1} U_{k}+\sum_{k=1}^{n q}(-1)^{k-1} V_{k} .
\end{aligned}
$$

where

$$
\begin{aligned}
& U_{k} \\
& =n \sum_{t_{1}+t_{2}+\cdots+t_{q}=k, 0 \leq t_{i} \leq q} \frac{q !}{t_{1} ! t_{2} ! \cdots t_{q} !(q-k) !} q^{(q-k) q^{n-1}} \\
& =\frac{n q !}{(q-k) !} q^{(q-k) q^{n-1}} \sum_{t_{1}+t_{2}+\cdots+t_{q}=k, 0 \leq t_{i} \leq q} \frac{1}{t_{1} ! t_{2} ! \cdots t_{q} !}
\end{aligned}
$$

and

$$
V_{k}=q \sum_{k_{1}+\cdots+k_{n}=k, 0 \leq k_{i} \leq k-1,0 \leq k_{i} \leq q}\left(\prod_{j=1}^{n} C\left(q, k_{j}\right)\right) q^{\prod_{j=1}^{n}\left(q-k_{j}\right)}
$$

Proof: Let

$$
S=\{\langle i: a: b\rangle \mid a, b \in Q, i \in[n]\} .
$$

We have

$$
\langle-:-:-\rangle=\bigcup_{i \in[n]} \bigcup_{a \in Q} \bigcup_{b \in Q}\langle i: a: b\rangle
$$

and $|\langle-:-:-\rangle|=\sum_{k=1}^{n q^{2}}(-1)^{k-1} N_{k}$, where

$$
N_{k}=\sum_{s \in S,|s|=k}\left|\bigcap_{T \in S} T\right| .
$$

We write all the $n q^{2}$ elements of $S$ as the following $n$ matrices. 


$$
\begin{gathered}
M_{1}=\left(\begin{array}{cccc}
\left\langle 1: a_{1}: b_{1}\right\rangle & \left\langle 1: a_{1}: b_{2}\right\rangle & \cdots & \left\langle 1: a_{1}: b_{q}\right\rangle \\
\left\langle 1: a_{2}: b_{1}\right\rangle & \left\langle 1: a_{2}: b_{2}\right\rangle & \cdots & \left\langle 1: a_{2}: b_{q}\right\rangle \\
\vdots & \vdots & . & \vdots \\
\left\langle 1: a_{q}: b_{1}\right\rangle & \left\langle 1: a_{q}: b_{2}\right\rangle & \cdots & \left\langle 1: a_{q}: b_{q}\right\rangle
\end{array}\right) \\
M_{2}=\left(\begin{array}{cccc}
\left\langle 2: a_{1}: b_{1}\right\rangle & \left\langle 2: a_{1}: b_{2}\right\rangle & \cdots & \left\langle 2: a_{1}: b_{q}\right\rangle \\
\left\langle 2: a_{2}: b_{1}\right\rangle & \left\langle 2: a_{2}: b_{2}\right\rangle & \cdots & \left\langle 2: a_{2}: b_{q}\right\rangle \\
\vdots & \vdots & \ddots & \vdots \\
\left\langle 2: a_{q}: b_{1}\right\rangle & \left\langle 2: a_{q}: b_{2}\right\rangle & \cdots & \left\langle 2: a_{q}: b_{q}\right\rangle
\end{array}\right) \\
M_{n}=\left(\begin{array}{cccc}
\left\langle n: a_{1}: b_{1}\right\rangle & \left\langle n: a_{1}: b_{2}\right\rangle & \cdots & \left\langle n: a_{1}: b_{q}\right\rangle \\
\left\langle n: a_{2}: b_{1}\right\rangle & \left\langle n: a_{2}: b_{2}\right\rangle & \cdots & \left\langle n: a_{2}: b_{q}\right\rangle \\
\vdots & \vdots & \ddots & \vdots \\
\left\langle n: a_{q}: b_{1}\right\rangle & \left\langle n: a_{q}: b_{2}\right\rangle & \cdots & \left\langle n: a_{q}: b_{q}\right\rangle
\end{array}\right)
\end{gathered}
$$

We combine all the above $M_{i}$ to form a $n q \times q$ matrix $M$ whose first $q$ rows are $M_{1}$, the second $q$ rows are $M_{2}, \cdots$, the last $q$ rows are $M_{n}$. In other words, we have

$$
M=\left(\begin{array}{cccc}
\left\langle 1: a_{1}: b_{1}\right\rangle & \left\langle 1: a_{1}: b_{2}\right\rangle & \cdots & \left\langle 1: a_{1}: b_{q}\right\rangle \\
\left\langle 1: a_{2}: b_{1}\right\rangle & \left\langle 1: a_{2}: b_{2}\right\rangle & \cdots & \left\langle 1: a_{2}: b_{q}\right\rangle \\
\vdots & \vdots & \ddots & \vdots \\
\left\langle 1: a_{q}: b_{1}\right\rangle & \left\langle 1: a_{q}: b_{2}\right\rangle & \cdots & \left\langle 1: a_{q}: b_{q}\right\rangle \\
\left\langle 2: a_{1}: b_{1}\right\rangle & \left\langle 2: a_{1}: b_{2}\right\rangle & \cdots & \left\langle 2: a_{1}: b_{q}\right\rangle \\
\left\langle 2: a_{2}: b_{1}\right\rangle & \left\langle 2: a_{2}: b_{2}\right\rangle & \cdots & \left\langle 2: a_{2}: b_{q}\right\rangle \\
\vdots & \vdots & \ddots & \vdots \\
\left\langle 2: a_{q}: b_{1}\right\rangle & \left\langle 2: a_{q}: b_{2}\right\rangle & \cdots & \left\langle 2: a_{q}: b_{q}\right\rangle \\
\vdots & \vdots & \ddots & \vdots \\
\left\langle n: a_{1}: b_{1}\right\rangle & \left\langle n: a_{1}: b_{2}\right\rangle & \cdots & \left\langle n: a_{1}: b_{q}\right\rangle \\
\left\langle n: a_{2}: b_{1}\right\rangle & \left\langle n: a_{2}: b_{2}\right\rangle & \cdots & \left\langle n: a_{2}: b_{q}\right\rangle \\
\vdots & \vdots & \ddots & \vdots \\
\left\langle n: a_{q}: b_{1}\right\rangle & \left\langle n: a_{q}: b_{2}\right\rangle & \cdots & \left\langle n: a_{q}: b_{q}\right\rangle
\end{array}\right)
$$

We are going to choose $k$ elements from $M$ to form the intersection. In order to get a possible non empty intersection, we know all these $k$ elements must come from either the same $M_{i}$ (for some fixed $i$ ) or all of them from the same column of $M$ by Proposition 2.3 .

Each $M_{i}$ is in fact the transpose of $B$ and each column of $M$ is all the elements of $A$ (As sets, they are equal). Hence, a typical intersection is either the one in Theorem 3.9 or the one in Theorem 3.12. But these two cases are not disjoint.

Suppose we choose $k_{i}$ elements from

$$
\begin{aligned}
& M_{i}, i=1,2, \cdots, n, k_{1}+k_{2}+\cdots+k_{n}=k, \\
& 0 \leq k_{i} \leq k, i=1,2, \cdots, n
\end{aligned}
$$

If there exist $i$ such that $k_{i}=k$, then $k_{j}=0, \forall j \neq i$. This implies the intersection looks like the one in Lemma 3.11 and $k \leq q$.

If $0 \leq k_{i} \leq k-1, \forall i \in[n]$, then the intersection looks like the one in Lemma 3.8 and $k \leq n q$.

The above two cases are disjoint now. By Lemma 3.11 and Lemma 3.8, we get

$$
\begin{aligned}
N_{k} & =\sum_{s \subset S,|s|=k}\left|\bigcap_{T \in S} T\right|=\sum_{k_{1}+\cdots+k_{n}=k, 0 \leq k_{i} \leq k} \\
& =\sum_{\exists i, k_{i}=k}+\sum_{k_{i} \leq k-1, i=1, \cdots, n}=U_{k}+V_{k}
\end{aligned}
$$

where (Note: there are $n$ matrices $M_{1}, M_{2}, \cdots, M_{n}$ and $q$ columns of $M$ )

$$
\begin{aligned}
U_{k} & =n \sum_{t_{1}+t_{2}+\cdots+t_{q}=k, 0 \leq t_{i} \leq q} \frac{q !}{t_{1} ! t_{2} ! \cdots t_{q} !(q-k) !} q^{(q-k) q^{n-1}} \\
& =\frac{n q !}{(q-k) !} q^{(q-k) q^{n-1}} \sum_{t_{1}+t_{2}+\cdots+t_{q}=k, 0 \leq t_{i} \leq q} \frac{1}{t_{1} ! t_{2} ! \cdots t_{q} !} \\
V_{k} & =\sum_{k_{1}+\cdots+k_{n}=k, 0 \leq k_{i} \leq k-1,0 \leq k_{i} \leq q}\left(\prod_{j=1}^{n} C\left(q, k_{j}\right)\right) q^{\prod_{j=1}^{n}\left(q-k_{j}\right)}
\end{aligned}
$$

Hence,

$$
\begin{aligned}
& |\langle-:-:-\rangle|=\sum_{k=1}^{n q^{2}}(-1)^{k-1} N_{k} \\
& =\sum_{k=1}^{n q^{2}}(-1)^{k-1}\left(U_{k}+V_{k}\right)=\sum_{k=1}^{q}(-1)^{k-1} U_{k}+\sum_{k=1}^{n q}(-1)^{k-1} V_{k},
\end{aligned}
$$

In the following, we will reduce the formula

$$
|\langle-:-:-\rangle|
$$

when $q=2$ and compare it with the one in [1]. We have

$$
|\langle-:-:-\rangle|=\sum_{k=1}^{2}(-1)^{k-1} U_{k}+\sum_{k=1}^{2 n}(-1)^{k-1} V_{k} .
$$

where

$$
\begin{aligned}
& U_{k}=n \sum_{t_{1}+t_{2}=k, 0 \leq t_{i} \leq 2} \frac{2 !}{t_{1} ! t_{2} !(2-k) !} 2^{(2-k) 2^{n-1}} \\
& V_{k}=2 \sum_{k_{1}+\cdots+k_{n}=k, 0 \leq k_{i} \leq k-1,0 \leq k_{i} \leq 2}\left(\prod_{j=1}^{n} C\left(2, k_{j}\right)\right) 2^{\prod_{j=1}^{n}\left(2-k_{j}\right)}
\end{aligned}
$$

A simple calculation shows that

$$
U_{1}=4 n 2^{2^{n-1}}=C(n, 1) 2^{2} 2^{2^{n-1}}
$$

and 


$$
U_{2}=4 n \text {. }
$$

$V_{1}=0$ since the condition of the sum is not satisfied.

$$
\begin{aligned}
V_{2} & =2 \sum_{k_{1}+\cdots+k_{n}=2,0 \leq k_{i} \leq 1}\left(\prod_{j=1}^{n} C\left(2, k_{j}\right)\right) 2^{\prod_{j=1}^{n}\left(2-k_{j}\right)} \\
& =C(n, 2) 2^{3} 2^{2^{n-2}}
\end{aligned}
$$

Note, $C(n, 2)$ is the number of solutions of the equation $k_{1}+\cdots+k_{n}=2,0 \leq k_{i} \leq 1$.

When $3 \leq k \leq 2 n$,

$$
\begin{aligned}
& V_{k}=2 \sum_{k_{1}+\cdots+k_{n}=k, 0 \leq k_{i} \leq 2}\left(\prod_{j=1}^{n} C\left(2, k_{j}\right)\right) 2^{\prod_{j=1}^{n}\left(2-k_{j}\right)} \\
& =C(n, k) 2^{k+1} 2^{2^{n-k}}+\sum_{1 \leq t \leq\left\lfloor\frac{k}{2}\right\rfloor} C(n, t) C(n-t, k-2 t) 2^{k-2 t+1}
\end{aligned}
$$

Note, $C(n, t) C(n-t, k-2 t)$ is the number of solutions of the equation $k_{1}+\cdots+k_{n}=2,0 \leq k_{i} \leq 2$ with exactly $t$ components equal to 2 .

hence, when $q=2$,

$$
\begin{aligned}
& |\langle-:-:-\rangle| \\
& =-4 n+\sum_{1 \leq k \leq n}(-1)^{k+1} C(n, k) 2^{k+1} 2^{2^{n-k}} \\
& +\sum_{3 \leq k \leq 2 n}(-1)^{k-1} \sum_{1 \leq t \leq\left\lfloor\frac{k}{2}\right\rfloor} C(n, t) C(n-t, k-2 t) 2^{k-2 t+1}
\end{aligned}
$$

When $n=1,2,3,4$, one can obtain (without calculator) the sequence $4,14,120,3514$. These results are consistent with those in [1]. By [1], the cardinality of $|\langle-:-:-\rangle|$ should be

$$
\begin{aligned}
& |\langle-:-:-\rangle| \\
& =2\left((-1)^{n}-n\right)+\sum_{1 \leq k \leq n}(-1)^{k+1} C(n, k) 2^{k+1} 2^{2^{n-k}} .
\end{aligned}
$$

So, we obtain the following combinatorial identity(for any positive integer $n$ ).

$$
\begin{aligned}
& \sum_{3 \leq k \leq 2 n}(-1)^{k-1} \sum_{1 \leq t \leq\left\lfloor\frac{k}{2}\right\rfloor} C(n, t) C(n-t, k-2 t) 2^{k-2 t+1} \\
& =2\left((-1)^{n}+n\right)
\end{aligned}
$$

The left sum should be explained as 0 if $n=1$. As usual, $C(n, k)$ is 0 if $k>n$.

From Theorem 3.1, we know $|\langle i: a: b\rangle|=q^{q^{n}-q^{n-1}}$, since $\langle-:-:-\rangle=\bigcup_{i \in[n]} \bigcup_{a \in \mathbb{F}} \bigcup_{b \in \mathbb{F}}\langle i: a: b\rangle$, we obtain

$$
|\langle-:-:-\rangle| \leq n q^{2} q^{(q-1) q^{n-1}} \text {. }
$$

In order to get an intuitive idea about the magnitude of all the cardinality numbers, We will find their asymptote as $n \rightarrow \infty$ or $q \rightarrow \infty$.
We have the following notation

Definition 3.14 We call $f(x) \stackrel{x}{\cong} g(x)$ if $\lim _{x \rightarrow \infty} \frac{f(x)}{g(x)}=1$.

Now, we can list all the cardinalities asymptotically.

Theorem 3.15 If $n \geq 4$ and $q \geq 2$, then

$$
\begin{aligned}
& |\langle i: a: b\rangle|=q^{(q-1) q^{n-1}} ;|\langle i: a:-\rangle|=q q^{(q-1) q^{n-1}} ; \\
& |\langle i:-: b\rangle| \cong q q^{(q-1) q^{n-1}},|\langle i:-: b\rangle| \stackrel{q}{\cong} q q^{(q-1) q^{n-1}} ; \\
& |\langle-: a: b\rangle| \stackrel{n}{\cong} n q^{(q-1) q^{n-1}},\langle-: a: b\rangle \mid \stackrel{q}{\cong} n q^{(q-1) q^{n-1}} ; \\
& |\langle-: a:-\rangle| \cong n q q^{(q-1) q^{n-1}},|\langle-: a:-\rangle| \stackrel{q}{\cong} n q q^{(q-1) q^{n-1}} ; \\
& |\langle-:-: b\rangle| \stackrel{n}{\cong} n q q^{(q-1) q^{n-1}},|\langle-:-: b\rangle| \stackrel{q}{\cong} n q q^{(q-1) q^{n-1}} ; \\
& |\langle i:-:-\rangle| \stackrel{n}{\cong} q^{2} q^{(q-1) q^{n-1}},|\langle i:-:-\rangle| \stackrel{q}{\cong} q^{2} q^{(q-1) q^{n-1}} ; \\
& |\langle-:-:-\rangle| \stackrel{n}{\cong} n q^{2} q^{(q-1) q^{n-1}},|\langle-:-:-\rangle| \stackrel{q}{\cong} n q^{2} q^{(q-1) q^{n-1}} .
\end{aligned}
$$

Proof:

The first two rows are Theorem 3.1 and Theorem 3.2.

We will give a proof of the last row, the others are similar and easier.

$$
\begin{aligned}
& |\langle-:-:-\rangle|=\sum_{k=1}^{q}(-1)^{k-1} U_{k}+\sum_{k=1}^{n q}(-1)^{k-1} V_{k} . \\
& U_{1}=\frac{n q !}{(q-1) !} q^{(q-1) q^{n-1}} \sum_{t_{1}+t_{2}+\cdots+t_{q}=1,0 \leq t_{i} \leq q} \frac{1}{t_{1} ! t_{2} ! \cdots t_{q} !} \\
& =n q^{2} q^{(q-1) q^{n-1}} .
\end{aligned}
$$

When $2 \leq k \leq q$, we have

$$
\begin{aligned}
& U_{k}=\frac{n q !}{(q-k) !} q^{(q-k) q^{n-1}} \sum_{t_{1}+t_{2}+\cdots+t_{q}=k, 0 \leq t_{i} \leq q} \frac{1}{t_{1} ! t_{2} ! \cdots t_{q} !} \\
& \leq n q ! q^{(q-2) q^{n-1}} \sum_{0 \leq t_{i} \leq q, i=1,2, \cdots, q} 1=n q ! q^{(q-2) q^{n-1}}(q+1)^{q} .
\end{aligned}
$$

Hence,

$$
\begin{aligned}
& \frac{\left|\sum_{k=2}^{q}(-1)^{k-1} U_{k}\right|}{U_{1}} \leq \frac{q n q !(q+1)^{q} q^{(q-2) q^{q-1}}}{n q^{2} q^{(q-1) q^{n-1}}} \leq \frac{q^{q-1}(q+1)^{q}}{q^{q^{n-1}}} \\
& \leq \frac{q^{q-1}(q)^{2 q}}{q^{q^{n-1}}} \leq \frac{q^{3 q}}{q^{q^{n-1}} .} \\
& \text { So, } \lim _{n \rightarrow \infty} \frac{\left|\sum_{k=2}^{q}(-1)^{k-1} U_{k}\right|}{U_{1}}=0 .
\end{aligned}
$$

$V_{1}=0$ since the condition of the sum is not satisfied. When $2 \leq k \leq n q$, we have 


$$
\begin{aligned}
V_{k} & =q \sum_{k_{1}+\cdots+k_{n}=k, 0 \leq k_{i} \leq k-1,0 \leq k_{i} \leq q}\left(\prod_{j=1}^{n} C\left(q, k_{j}\right)\right) q^{\prod_{j=1}^{n}\left(q-k_{j}\right)} \\
& \leq q \sum_{0 \leq k_{i} \leq q, i=1,2, \cdots, n}(q !)^{n} q^{(q-1)^{2} q^{n-2}} \\
& =q(q+1)^{n}(q !)^{n} q^{(q-1)^{2} q^{n-2} .}
\end{aligned}
$$

Note, $\prod_{j=1}^{n}\left(q-k_{j}\right) \leq(q-1)^{2} q^{n-2}$. Hence,

$$
\left|\sum_{k=1}^{n q}(-1)^{k-1} V_{k}\right| \leq n q q(q+1)^{n}(q !)^{n} q^{(q-1)^{2} q^{n-2}} .
$$

We obtain

$$
\begin{aligned}
& \frac{\left|\sum_{k=1}^{n q}(-1)^{k-1} V_{k}\right|}{U_{1}} \leq \frac{n q q(q+1)^{n}(q !)^{n} q^{(q-1)^{2} q^{n-2}}}{n q^{2} q^{(q-1) q^{n-1}}} \\
& =\frac{(q+1)^{n}(q !)^{n}}{q^{(q-1) q^{n-2}}} \leq \frac{(q+1)^{n} q^{q n}}{q^{(q-1) q^{n-2}}} \leq \frac{q^{(2+q) n}}{q^{q^{n-2}}} .
\end{aligned}
$$

Hence,

$$
\lim _{n \rightarrow \infty} \frac{\left|\sum_{k=1}^{n q}(-1)^{k-1} V_{k}\right|}{U_{1}}=0 .
$$

In summary, we obtain

$$
\lim _{n \rightarrow \infty} \frac{|\langle-:-:-\rangle|}{n q^{2} q^{(q-1) q^{n-1}}}=1 .
$$

In other words,

$$
|\langle-:-:-\rangle| \stackrel{n}{\cong} n q^{2} q^{(q-1) q^{n-1}} .
$$

From the above proof, it is also clear that we have

$$
\lim _{q \rightarrow \infty} \frac{|\langle-:-:-\rangle|}{n q^{2} q^{(q-1) q^{n-1}}}=1 .
$$

In other words,

$$
|\langle-:-:-\rangle| \stackrel{q}{\cong} n q^{2} q^{(q-1) q^{n-1}} .
$$

When $q=2$, the first equation of the last row in the above theorem has been obtained in [1].

\section{Conclusion}

In this paper, we generalized the definition of Boolean canalyzing functions to the functions of multi-state case. Using Inclusion and Exclusion Principle, we get formulas for the cardinality all such functions and the cardinalities of its various subsets. When $q=2$, we derive an interesting combinatorial identity by equating our formula to the one in [1]. Finally, for a better understanding to the magnitudes, we provide all the asymptotes of these cardinalities as either $n \rightarrow \infty$ or $q \rightarrow \infty$.

\section{Acknowledgements}

This work was initiated when the first and the third authors visited Virginia Bioinformatics Institute at Virginia Tech in June 2010. We thank Alan Veliz-Cuba and Franzeska Hinkelmann for many useful discussions. The first and the third authors thank Professor Reinhard Laubenbacher for his hospitality and for introducing them to Discrete Dynamical Systems. The third author was supported in part by a Research Initiation Program (RIP) award at Winston-Salem State University.

We greatly appreciate an anonymous reader. Because of his insightful comments, in this paper, the proofs for many lemmas are simplified, the results are more general (On any finite set instead of finite field).

\section{REFERENCES}

[1] W. Just, I. Shmulevich and J. Konvalina, "The Number and Probability of Canalyzing Functions," Physica D, Vol. 197, No. 3-4, 2004, pp. 211-221. doi:10.1016/j.physd.2004.07.002

[2] C. H. Waddington, "The Strategy of the Genes," George Allen and Unwin, London, 1957.

[3] R. Thomas and R. D'Ari, "Biological Feedback," CRC Press, Boca Raton, 1989.

[4] L. Steggles, et al., "Qualitatively Modelling and Analyzing Genetic Regulatory Networks: A Petri Net Approach," Bioinformatics, Vol. 23, No. 3, 2007, pp. 336-343. doi:10.1093/bioinformatics/bt1596

[5] M. Pogson, et al., "Formal Agent-Based Modelling of Intracellular Chemical Interactions," Biosystems, Vol. 85, No. 1, 2006, pp. 37-45. doi:10.1016/j.biosystems.2006.02.004 